\title{
ARTICLE
}

Clinical Study

\section{Alpha-fetoprotein kinetics in patients with hepatocellular carcinoma receiving ramucirumab or placebo: an analysis of the phase 3 REACH study}

Ian Chau (iD ${ }^{1}$, Joon Oh Park ${ }^{2}$, Baek-Yeol Ryoo ${ }^{3}$, Chia-Jui Yen $^{4}$, Ronnie Poon ${ }^{5}$, Davide Pastorelli ${ }^{6}$, Jean-Frédéric Blanc ${ }^{7}$, Masatoshi Kudo $^{8}$, Tulio Pfiffer ${ }^{9}$, Etsuro Hatano ${ }^{10}$, Hyun Cheol Chung ${ }^{11}$, Katerina Kopeckova ${ }^{12}$, Jean-Marc Phelip ${ }^{13}$, Giovanni Brandi ${ }^{14}$, Shinichi Ohkawa ${ }^{15}$, Chung-Pin Li ${ }^{16,17}$, Takuji Okusaka ${ }^{18}$, Yanzhi Hsu ${ }^{19}$, Paolo B. Abada ${ }^{20}$ and Andrew X. Zhu ${ }^{21}$

\begin{abstract}
BACKGROUND: Post-hoc analyses of AFP response and progression and their relationship with objective measures of response and survival were performed in patients from REACH.

METHODS: Serum AFP was measured at baseline and every 3 cycles (2 weeks/cycle). Associations between AFP and radiographic progression and efficacy end points were analysed.

RESULTS: Median percent AFP increase from baseline was smaller in the ramucirumab than in the placebo arm throughout treatment. Time to AFP progression (HR $0.621 ; P<0.0001)$ and to radiographic progression (HR $0.613 ; P<0.0001)$ favoured ramucirumab. Association between AFP and radiographic progression was shown at $6(\mathrm{OR} 6.44,95 \% \mathrm{Cl} 4.03,10.29 ; P<0.0001)$ and 12 weeks (OR 2.28, 95\% Cl 1.47, 3.53; $P=0.0002)$. AFP response was higher with ramucirumab compared with placebo $(P<0.0001)$. More patients in the ramucirumab arm experienced tumour shrinkage and AFP response compared with placebo. Survival was longer in patients with AFP response (13.6 months) than in patients without (6.2 months), irrespective of treatment (HR $0.457, P<$ 0.0001).

CONCLUSIONS: Treatment with ramucirumab prolonged time to AFP progression, slowed AFP increase and was more likely to induce AFP response. Similar benefits in radiographic progression and response correlated with AFP changes.
\end{abstract}

British Journal of Cancer https://doi.org/10.1038/s41416-018-0103-0

\section{INTRODUCTION}

Liver cancer is the sixth most commonly diagnosed cancer worldwide and the second most common cause of cancer death. ${ }^{1}$ Hepatocellular carcinoma (HCC) represents approximately $90 \%$ of primary liver cancers and occurs most frequently in patients with cirrhosis from chronic hepatitis B or C virus infection or alcohol abuse. $^{2}$

Alpha-fetoprotein (AFP) level has long been known to correlate with HCC prognosis and has historically played a role in diagnosis. Elevated AFP levels are associated with larger tumours, bilobar involvement, portal vein invasion, poorly differentiated histology and decreased median survival. ${ }^{3}$ Measurement of AFP level has been incorporated into some HCC prognostic scoring systems. ${ }^{4,5}$ While high levels of AFP are recognised as a poor prognostic factor, the utility of AFP response or progression during anticancer treatment is still unclear. There are limited studies in patients with HCC correlating AFP kinetics with treatment response during locoregional therapy or while on sorafenib and no published results of patients on second-line treatment.

Vascular endothelial growth factor (VEGF) is overexpressed in HCC and associated with poorer clinical outcomes, suggesting VEGF-mediated signalling is important in HCC pathogenesis and is

\footnotetext{
${ }^{1}$ Department of Medicine, Royal Marsden Hospital, Sutton, Surrey SM2 5PT, UK; ${ }^{2}$ Division of Hematology-Oncology, Department of Medicine, Samsung Medical Center, Sungkyunkwan University School of Medicine, Seoul 135-710, Korea; ${ }^{3}$ Department of Oncology, Asan Medical Center, University of Ulsan College of Medicine, Seoul 05505, Korea; ${ }^{4}$ Department of Internal Medicine, National Cheng Kung University Hospital, College of Medicine, National Cheng Kung University, Tainan 701 , Taiwan; ${ }^{5}$ Departmentof Surgery, The University of Hong Kong, Pokfulam, Hong Kong; ${ }^{6}$ Department of Oncology, Santa Maria del Prato Hospital, Feltre (Belluno) 32032, Italy; ${ }^{7}$ Department of HepatoGastroenterology and Medical Oncology, CHU de Bordeaux, Hôpital Haut-Lévêque, 33604 Pessac, France; ${ }^{8}$ Department of Gastroenterology and Hepatology, Kindai University

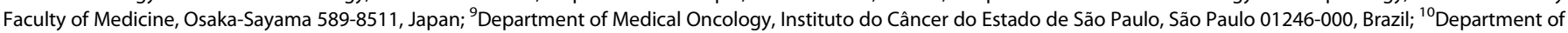
Surgery, Graduate School of Medicine, Kyoto University, Kyoto 606-8507, Japan; ${ }^{11}$ Department of Medical Oncology, Yonsei Cancer Center, Yonsei University College of Medicine, Seoul 03722, Korea; ${ }^{12}$ Department of Oncology, University Hospital Motol, 2nd Faculty of Medicine of Charles University, 15000 Praha, Czech Republic; ${ }^{13}$ Department of Gastroenterology and Digestive Oncology, University Hospital of St Etienne, 42100 Saint Etienne, France; ${ }^{14}$ Department of Experimental, Diagnostic and Specialty Medicine, University Hospital S. Orsola, 40138 Bologna, Italy; ${ }^{15}$ Division of Hepatobiliary and Pancreatic Oncology, Kanagawa Cancer Center, Yokohama $241-0815$, Japan; ${ }^{16}$ Division of Gastroenterology and Hepatology, Department of Medicine, Taipei Veterans General Hospital, Taipei 112, Taiwan; ${ }^{17}$ National Yang-Ming University School of Medicine, Taipei 112 ,

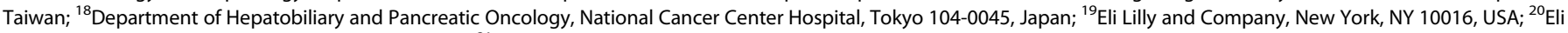
Lilly and Company, Indianapolis, IN 46285, USA and ${ }^{21}$ Department of Medicine, Harvard Medical School, Massachusetts General Hospital, Boston, MA 02114, USA Correspondence: lan Chau (lan.Chau@rmh.nhs.uk)

These authors contributed equally: Ian Chau, Andrew X. Zhu.
}

Received: 25 October 2017 Revised: 4 April 2018 Accepted: 11 April 2018

Published online: 29 May 2018 
a therapeutic target. ${ }^{6-8}$ Ramucirumab is a recombinant immunoglobulin G, subclass 1 monoclonal antibody that specifically binds to the extracellular domain of VEGFR-2 with high affinity, preventing binding of VEGF ligands and receptor activation. ${ }^{9}$ $\mathrm{REACH}$, a global, randomised, double-blinded placebo-controlled Phase 3 study, evaluated the efficacy and safety of single-agent ramucirumab for patients with advanced HCC after prior treatment with sorafenib $(N=565){ }^{10}$ Significant improvement in overall survival (OS) in the intent-to-treat (ITT) population was not achieved. However, a clinically meaningful improvement in OS was observed in patients with elevated baseline AFP levels ( $\geq 400$ $\mathrm{ng} / \mathrm{mL}[n=250])$ treated with ramucirumab vs placebo (OS 7.8 vs 4.2 months, respectively; hazard ratio (HR) $0.67, P=0.006)$.

In the REACH study, AFP values were collected at baseline and during treatment. Post-hoc analyses of AFP response and progression, and correlations with other measures of efficacy including time to progression (TTP), objective response rate (ORR), and OS, were performed.

\section{PATIENTS AND METHODS}

Patient selection, randomisation and masking

The details of eligibility for inclusion in the REACH trial were previously described. ${ }^{10}$

\section{Procedures}

Patients received either ramucirumab $8 \mathrm{mg} / \mathrm{kg}$ (ImClone Systems Corporation, Branchburg, NJ, USA) $(n=283)$ or placebo $(n=282)$ intravenously every 2 weeks until disease progression, unacceptable toxicity or withdrawal of consent. All patients received supportive care. Predefined dose modifications were allowed to manage treatment-related toxicity. ${ }^{10}$ Local radiological imaging was performed at baseline, every 6 weeks over the first 6 months of treatment and every 9 weeks thereafter. In the event of ramucirumab/placebo dose delays or missed doses, disease assessment and imaging studies were to be undertaken according to the original study schedule (i.e. every 6 weeks after first dose for the first 6 months and every 9 weeks thereafter), regardless of the actual number of on-study treatments received.

\section{Statistical definitions}

TTP was defined as the time from randomisation to radiographic progression; radiographic response was assessed by protocoldefined criteria based on Response Evaluation Criteria in Solid Tumors 1.1 (appendix); ORR was defined as the proportion of patients who achieved complete response (CR) or partial response $(\mathrm{PR})$ as their best overall response (BOR).

OS was defined as the time from randomisation to death from any cause.

Serum AFP levels were measured locally at baseline (within 2 weeks prior to randomisation), and every 3 cycles, i.e. every 6 weeks until treatment discontinuation, and at short-term followup. AFP progression was defined as $\geq 20 \%$ increase from non-zero baseline and absolute increase $\geq 10 \mathrm{ng} / \mathrm{mL}$. For the small number of patients $(n=4 ; 2$ in the ramucirumab arm, 2 in the placebo arm) with a true baseline AFP of zero, AFP progression was defined as absolute increase AFP $\geq 10 \mathrm{ng} / \mathrm{mL}$ from zero baseline. These definitions were chosen to limit the risk of non-significant variations in AFP levels being considered AFP progression. AFP response was assessed in the population subset with baseline AFP $\geq 1.5$ upper limit of normal (ULN) and was defined as $\geq 20 \%$ decrease from baseline. This threshold of a minimum level of baseline AFP was selected to allow for a meaningful analysis since patients with very low levels of baseline AFP experiencing nonsignificant variations in AFP levels during treatment could result in large percent changes. Changes of 20 and $50 \%$ from baseline have been examined in previous studies. ${ }^{11-13}$
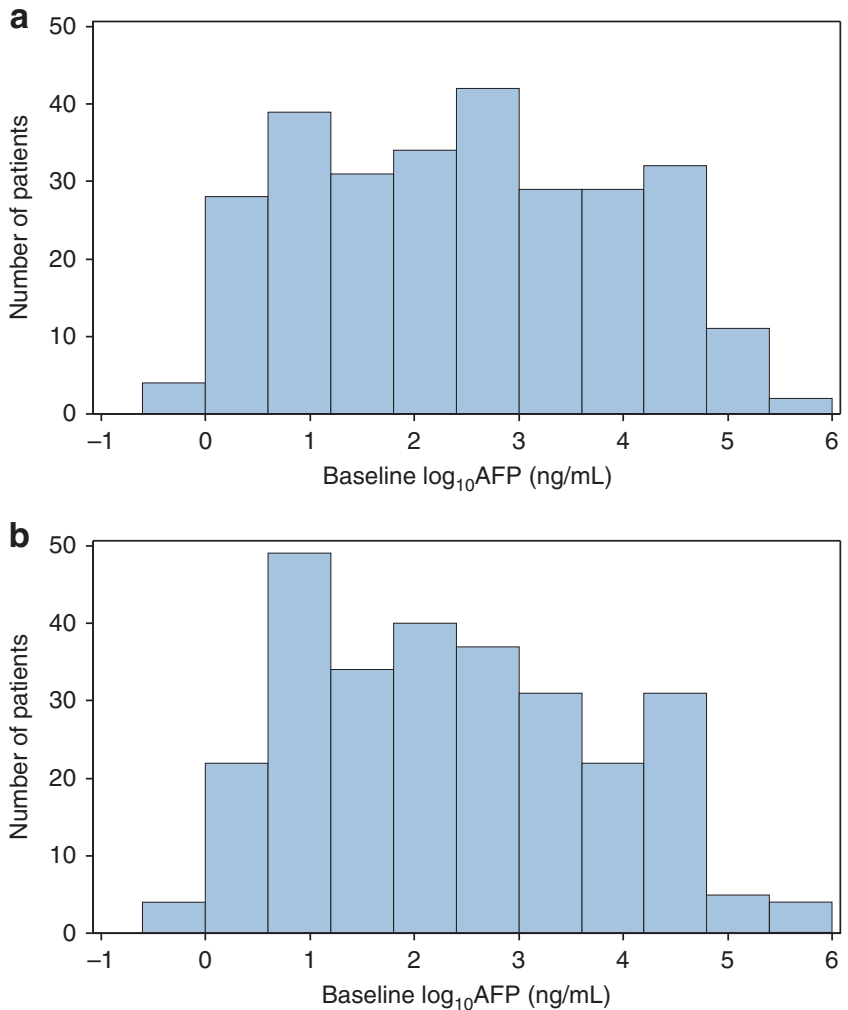

Fig. 1 Distribution of patients by baseline AFP level in each arm. a Patients treated with placebo and best standard of care. $\mathbf{b}$ Patients treated with ramucirumab and best standard of care. AFP alphafetoprotein

Statistical analysis

This post hoc analysis was conducted within the ITT population of REACH. The baseline distribution of patients by AFP level was plotted for comparison between arms. After taking log10 of baseline AFP values, the frequency (patient count) was plotted for each arm. AFP response rate is presented with $95 \%$ confidence interval (CI) and was compared using the Cochran-Mantel-Haenszel test. Percent change in AFP from baseline was analysed for each arm at each time point up to Cycle 12. Analyses evaluated the association between the events of AFP progression and radiographic progression in each AFP level measurement time interval (Fisher's exact test and odds ratio [OR]).

Time to AFP progression and time to radiographic progression between treatment arms were evaluated by the Kaplan-Meier method and tested by a stratified log-rank test. HR was generated using a stratified Cox proportional hazard model. AFP response rate is presented with $95 \% \mathrm{Cl}$ and compared using Cochran-Mantel-Haenszel test. The statistical analysis was done using the $\mathrm{SAS}^{\circledR}$ software Version 9.2.

AFP percent changes observed in patients in the ramucirumab arm were compared to those in the placebo arm at Cycles 3, 6, 9 and 12 by non-parametric Wilcoxon rank-sum tests.

\section{RESULTS}

Baseline patient and disease characteristics in the ITT population were well balanced between treatment groups. ${ }^{10}$ Baseline characteristics of patients in whom AFP response was assessed (with baseline AFP above 1.5 ULN, $n=417$ ) were also well balanced and, apart from baseline, AFP showed no meaningful differences from the baseline characteristics of the ITT population 
a

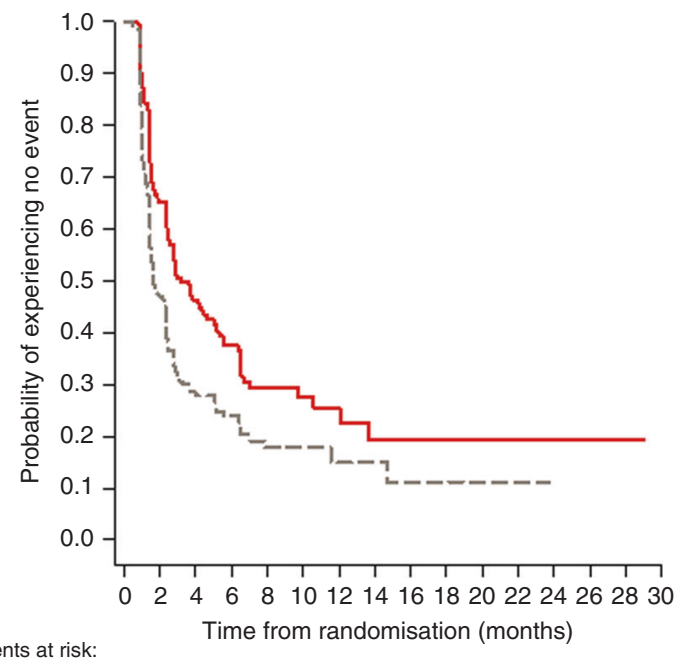

Patients at risk:

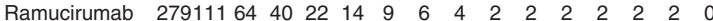
$\begin{array}{lllllllllllllllll}\text { Placebo } & 281 & 88 & 38 & 21 & 12 & 9 & 5 & 4 & 3 & 1 & 1 & 1 & 0 & 0 & 0 & 0\end{array}$

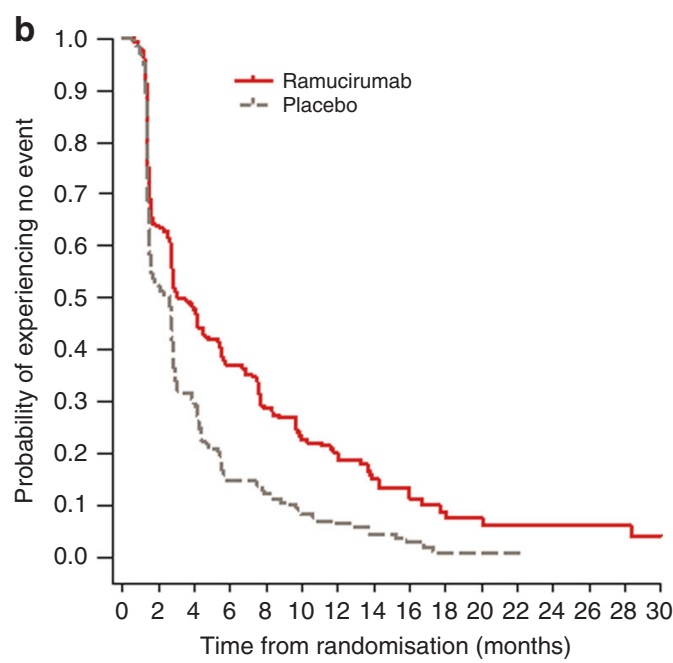

$28315610870 \begin{array}{lllllllllllll}51 & 37 & 29 & 19 & 12 & 7 & 6 & 4 & 3 & 3 & 3 & 1\end{array}$

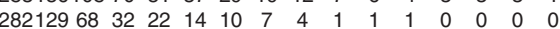

\begin{tabular}{lc|c|c|c}
\hline & \multicolumn{2}{c|}{ Time to AFP progression ${ }^{\mathrm{a}}$} & \multicolumn{2}{c}{ Time to radiographic progression } \\
\hline & $\begin{array}{c}\text { Ramucirumab } \\
N=279\end{array}$ & $\begin{array}{c}\text { Placebo } \\
N=281\end{array}$ & $\begin{array}{c}\text { Ramucirumab } \\
N=283\end{array}$ & $\begin{array}{c}\text { Placebo } \\
N=282\end{array}$ \\
\cline { 2 - 4 } Median, months $(95 \% \mathrm{Cl})$ & $3.2(2.7,4.6)$ & $1.6(1.5,2.3)$ & $3.5(2.8,4.5)$ & $2.6(1.6,2.8)$ \\
\hline HR $(95 \% \mathrm{Cl})$ & \multicolumn{2}{c}{$0.621(0.495,0.780)$} & \multicolumn{2}{c}{$0.613(0.505,0.744)$} \\
\hline$P$-value (log-rank) & \multicolumn{2}{c}{$<0.0001$} & $<.0001$ \\
\hline
\end{tabular}

Fig. 2 Kaplan-Meier plots of $\mathbf{a}$ time to AFP progression ${ }^{\mathbf{a}}$ and $\mathbf{b}$ time to radiographic progression in the ITT population. AFP alpha-fetoprotein, $\mathrm{Cl}$ confidence interval, HR hazard ratio, ITT intention to treat. ${ }^{a}$ Five patients were not included because of missing baseline AFP level

(Table S1). Additional details on the REACH study population have been disclosed previously. ${ }^{10}$ After log transformation of baseline AFP, the distribution of patients by log AFP for each treatment arm appeared similar in both treatment arms. A $t$-test on the logtransformed baseline AFP levels did not show any significant difference in baseline AFP levels between two arms (Fig. 1).

Changes in AFP relative to baseline were analysed and defined as either AFP progression or response, or neither, as per the Patients and methods section. A Kaplan-Meier plot of time to AFP progression for patients treated with ramucirumab vs placebo is shown in Fig. 2. The median time to AFP progression was 3.2 months in the ramucirumab arm $(95 \% \mathrm{Cl} 2.7,4.6, n=279)$ and 1.6 months in the placebo arm $(95 \% \mathrm{Cl} 1.5,2.3, n=281)$ with an HR of $0.621(P<0.0001)$.

Consistent with the results on time to AFP progression, patients treated with ramucirumab were more likely to experience an AFP response (decrease) at any time post-baseline compared to patients treated with placebo (ramucirumab: $27.8 \%$ vs placebo: $10.8 \% ; P<0.0001)$ and less likely to experience AFP progression (increase) at any time post-baseline compared to those treated with placebo (ramucirumab: $62.4 \%$ vs placebo: $75.9 \% ; P=0.0033$ ). The difference in the percentage of patients with AFP response or progression between arms was significant when AFP response and progression were defined as a $20 \%(P<0.0001)$ or $50 \%(P=$ $0.0004)$ change from baseline. No meaningful differences in the rates of AFP response were observed for patients with baseline $\geq 400 \mathrm{ng} / \mathrm{mL}$ compared to those with AFP $<400 \mathrm{ng} / \mathrm{mL}$, suggesting that AFP response was independent of the magnitude of baseline AFP (data not shown).

Waterfall plots of best percent change in AFP from baseline for patients treated with ramucirumab or placebo also support the results of the analyses on AFP response and progression (Fig. 3a). The proportion of patients who experienced an increase in AFP was not only lower in the ramucirumab arm but the magnitude of the increase also appeared smaller when compared with the placebo arm. Of note, 23 patients on the placebo arm also experienced an AFP response. An assessment of baseline characteristics for these patients did not identify any meaningful differences from the rest of the cohort, and other definitions of AFP response would not eliminate the presence of patients with AFP response in the placebo arm and likely represent true spontaneous responses.

To further assess the kinetics of AFP during treatment, AFP percent changes from baseline were calculated, and the median percent change from baseline evaluated by treatment arm (Fig. 4a). At each AFP assessment time point following baseline at Cycles 3, 6, 9 and 12, the median percent increase in AFP level from baseline was smaller in the ramucirumab arm $(4,0,3,33 \%)$ than in the placebo arm $(37,50,99,78 \%)$, respectively, and AFP percent change was significantly smaller in the ramucirumab arm at Cycles 3, 6 and 9.

Correlation of AFP changes with measures of radiographic response or progression

Kaplan-Meier plots of time to AFP progression and time to radiographic progression were similar in appearance (Fig. 2). The median time to radiographic progression was 3.5 months on the ramucirumab arm $(95 \% \mathrm{Cl} 2.8,4.5, n=283)$ and 2.6 months in the placebo arm (95\% Cl 1.6, 2.8, $n=282$, HR 0.613, $P<0.0001)$. A high association between AFP progression and radiographic progression occurring within each tumour assessment period was also observed (OR 6.4, 95\% Cl 4.0, 10.3, $P<0.0001$ for up to Week 6, OR 2.3, 95\% Cl 1.5, 3.5, $P=0.0002$ for Weeks 6-12) (Table 1).

Median percent change in AFP was further assessed in subgroups of patients defined by their best overall radiographic response (objective response [complete response/partial response $(\mathrm{CR} / \mathrm{PR})]$, disease control [CR/PR/stable disease (SD)] and progressive disease [PD]). For patients with a best overall radiographic 


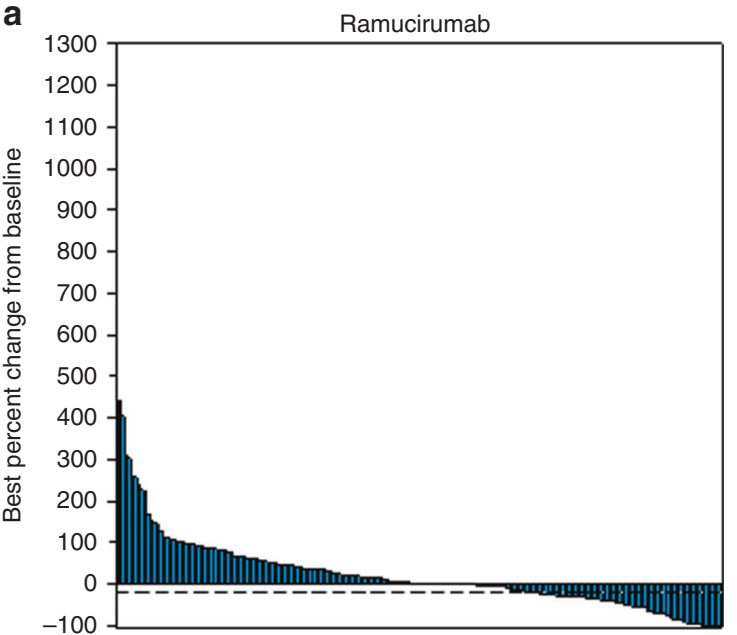

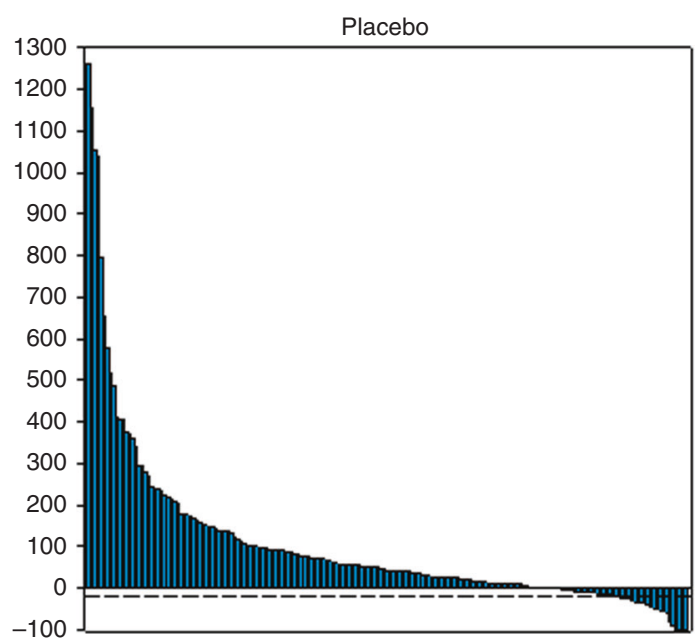

Patients with no AFP response

Patients with AFP response

b

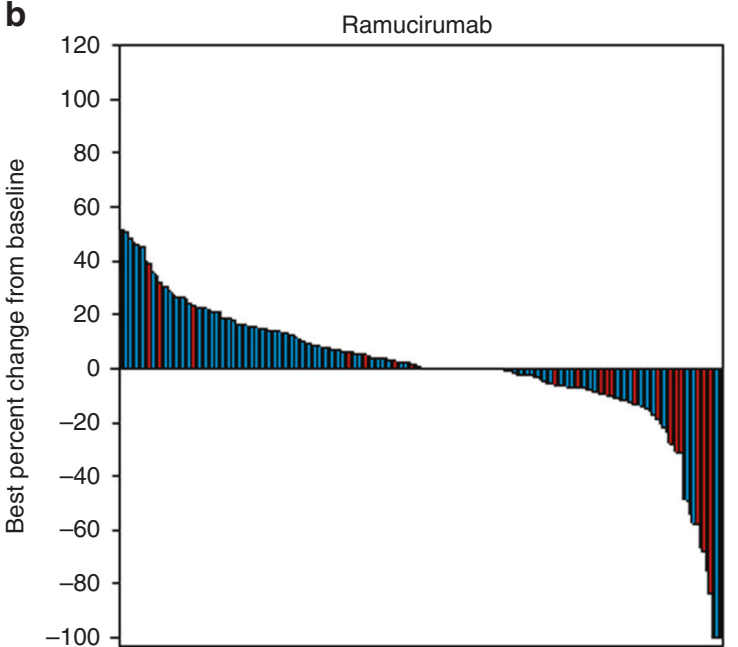

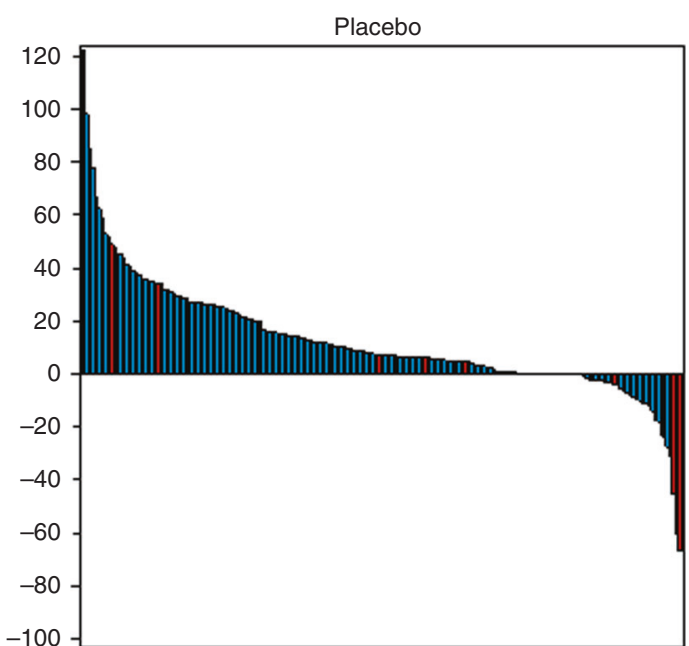

Fig. 3 Waterfall plots of response for patients by treatment arm. a Best percent change in AFP from baseline measurements by treatment arm. b Best percent change in radiographic tumour response, and relationship with AFP response. AFP alpha fetoprotein

response of $C R / P R$, the observed median percent change in AFP was a decrease in both treatment arms, with more patients in the ramucirumab arm experiencing an objective response compared to placebo (Fig. 4b). However, this should to be interpreted with caution given the small number of patients with best response of $\mathrm{CR} / \mathrm{PR}$ and the differences between the groups were not statistically significant (Fig. 4b). For patients with a BOR of disease control (CR/PR/SD), the median percent AFP increase from baseline for patients in the ramucirumab arm was lower than what was observed in the placebo arm at each cycle (Fig. 4c), with AFP percent changes being statistically different for the two arms at Cycles 6 and 9. In patients experiencing a best response of PD defined by radiographic progression, AFP increase from baseline for patients on the ramucirumab arm was significantly lower than what was observed on the placebo arm at Cycle 3 (Fig. 4d). There was no data available at Cycle 9 or 12 for this subgroup of patients as most patients with a best response of progression had already discontinued treatment.

Waterfall plots of radiographic tumour response by treatment arm and the relationship with AFP response (yes vs no) are shown in Fig. 3b. A higher proportion of patients experienced a radiographic response in the ramucirumab arm compared with the placebo arm. Most patients with a radiographic response (14 on RAM, 4 on PBO) also experienced an AFP response (10 on RAM, 3 on $\mathrm{PBO}$ ).

Overall survival by AFP response

Additional analyses on the relationship between AFP response and OS were performed. A Kaplan-Meier plot of OS for patients (baseline AFP $>1.5 \times U L N$ ), irrespective of treatment arm, with either an AFP response $(n=80)$ or no AFP response $(n=337)$ is shown in Fig. 5a. The median OS for patients with an AFP response was significantly longer than that for patients without AFP response (13.6 vs 6.2 months, $\mathrm{HR}=0.457,95 \% \mathrm{Cl} 0.338, .616 ; P$ $<0.0001)$.

Kaplan-Meier plots of OS by treatment arm in patients with either an AFP response (Fig. 5b) or no AFP response (Fig. 5c) are shown in Fig. 5. In patients with an AFP response, there was no statistically significant survival benefit of ramucirumab treatment over placebo over the course of treatment (up to 28 months). Notably, in patients without an AFP response, a potentially significant survival benefit was observed for patients treated with ramucirumab compared to placebo (7.2 vs 5.2 months, $\mathrm{HR}=0.758$, $95 \% \mathrm{Cl} 0.600,0.958 ; P=0.020)$, suggesting that even patients with elevated AFP $(>1.5 \times$ ULN) who do not have an AFP response may derive a benefit from ramucirumab treatment. 


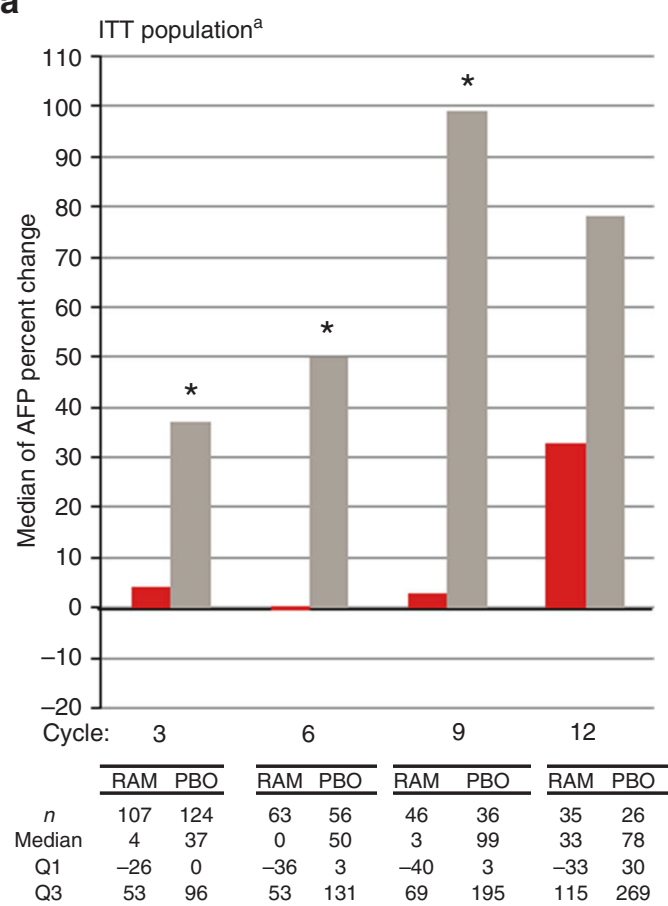

C

ITT population ${ }^{\mathrm{a}}$ and best overall response of $\mathrm{CR} / \mathrm{PR} / \mathrm{SD}$

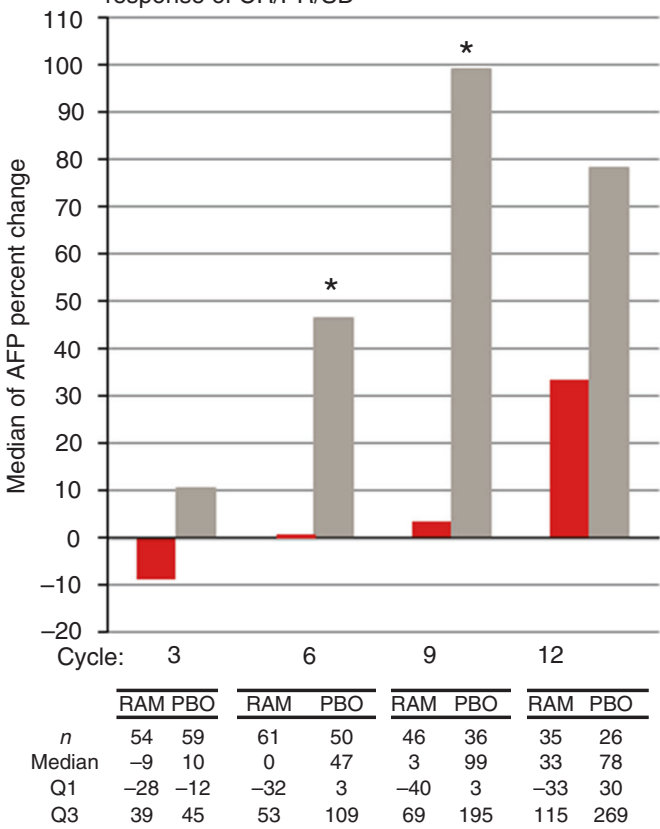

b

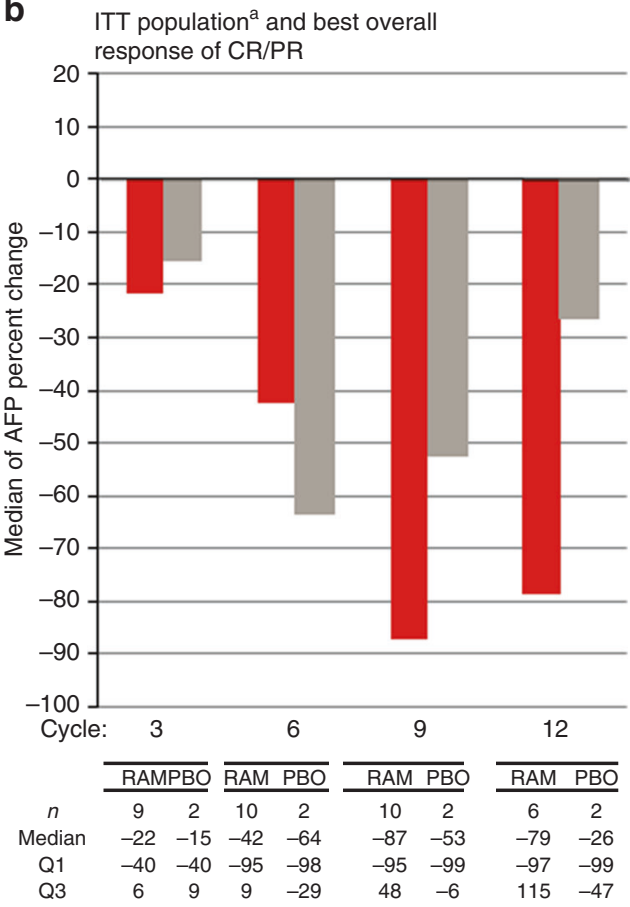

d

ITT population ${ }^{\mathrm{a}}$ and best overall

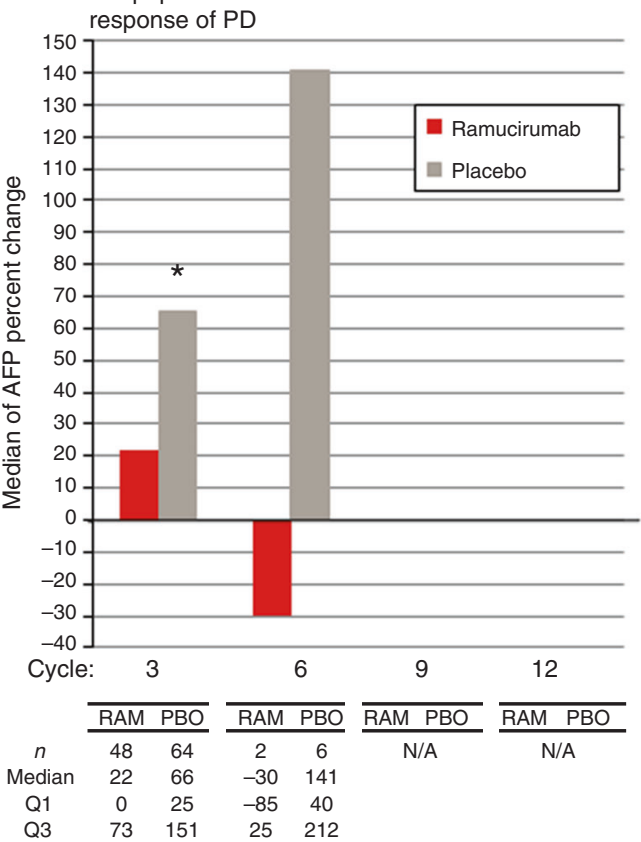

Fig. 4 AFP percent change from baseline by cycle. Medians of AFP percent changes from baseline were plotted every three cycles for patients from the ITT population with baseline AFP $\geq 1.5$ ULN by treatment arm. $\mathbf{a}$ for all patients; $\mathbf{b}$ for patients with best overall response of CR/PR; $\mathbf{c}$ for patients with best overall response of CR/PR/SD; $\mathbf{d}$ for patients with best overall response of PD. aITT population with baseline AFP $\geq 1.5$ ULN. *Indicates a statistical difference between the two groups by non-parametric Wilcoxon rank-sum tests. AFP alpha-fetoprotein, CR complete response, ITT intention to treat, NA not available, PD progressive disease, PR partial response, SD stable disease, Q1 lower quartile, Q3 upper quartile

Further analyses showed that 11 patients completely normalised their AFP level, 8 from the ramucirumab arm, and 3 from the placebo arm. The OS for these 11 patients who completely normalised their AFP level was significantly longer than the OS for patients who had AFP response without completely normalising their AFP level $(n=111)$ (25.6 vs 10.6 months, respectively, $\mathrm{HR}=$ $0.147, P=0.0019)$.

\section{DISCUSSION}

Serum AFP has long been recognised as both a diagnostic and prognostic marker. ${ }^{14-17}$ However, assessing AFP kinetics during treatment has been limited. Some retrospective studies have been performed in patients undergoing locoregional therapy, where an AFP response has been associated with a longer survival following transarterial chemoembolisation. ${ }^{12,18,19}$ In the more advanced 
Table 1. Radiographic progression and AFP progression by tumour measurement period

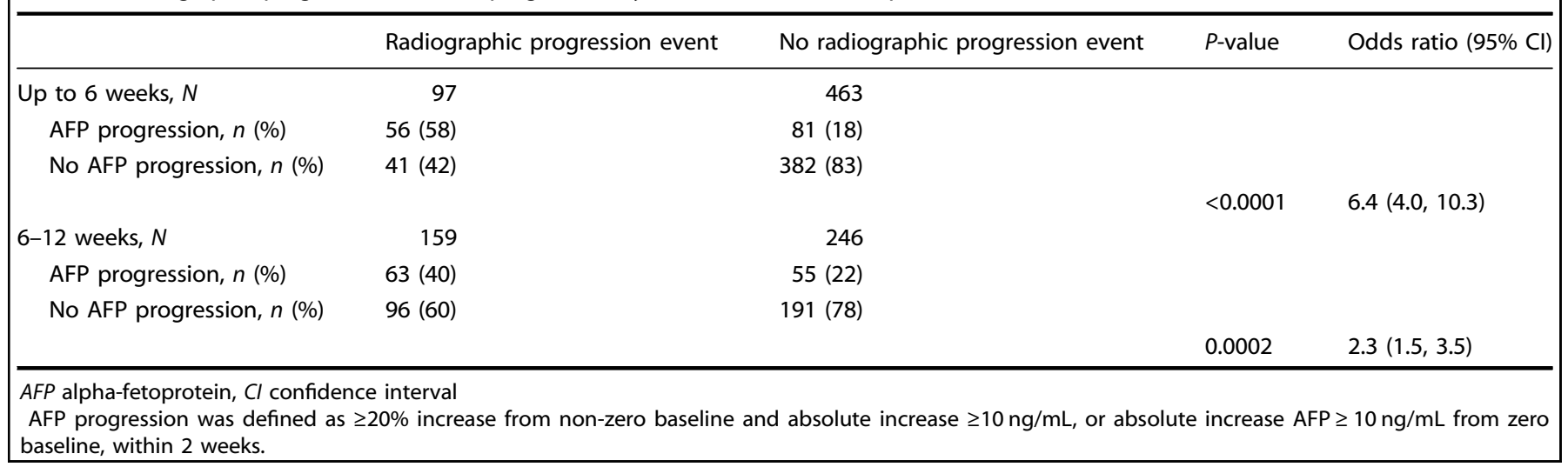

a

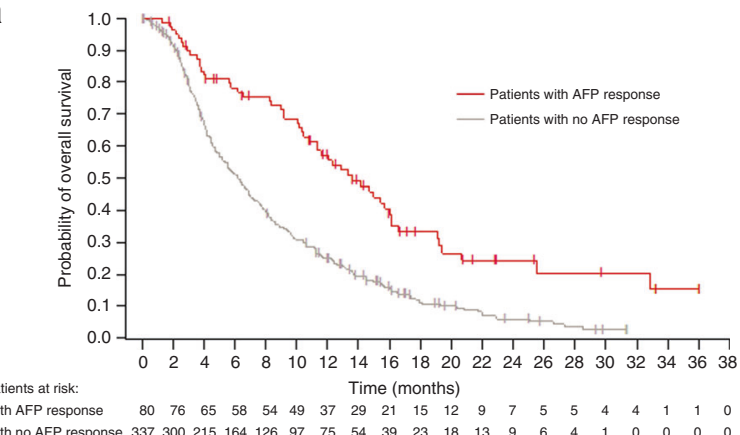

C

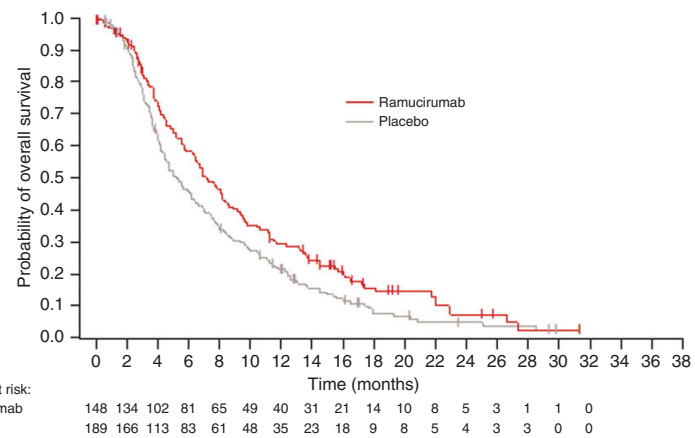

b

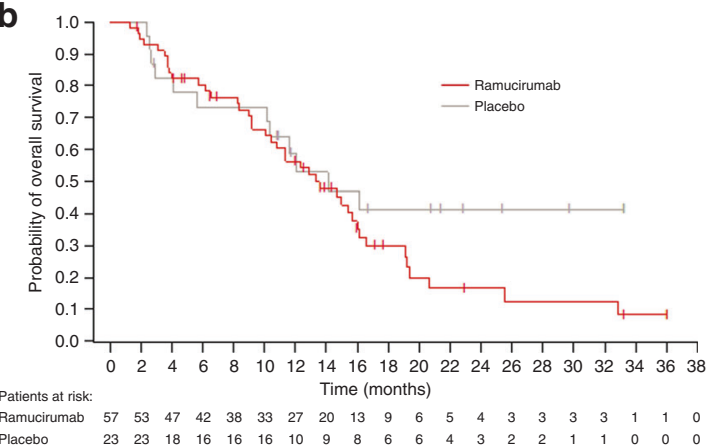

\begin{tabular}{|c|c|c|c|c|c|c|}
\hline & \multicolumn{6}{|c|}{ Overall survival in patients with } \\
\hline & \multicolumn{2}{|c|}{ AFP response } & \multicolumn{2}{|c|}{ AFP response } & \multicolumn{2}{|c|}{ No AFP response } \\
\hline & Yes & No & RAM & PBO & RAM & PBO \\
\hline & $n=80$ & $n=337$ & $n=57$ & $n=23$ & $n=148$ & $n=189$ \\
\hline Median, months $(95 \% \mathrm{Cl})$ & 13.6 & 6.2 & 13.4 & 14.1 & 7.2 & 5.2 \\
\hline $\mathrm{HR}(95 \% \mathrm{Cl})$ & \multicolumn{2}{|c|}{$0.457(0.338,0.616)$} & \multicolumn{2}{|c|}{$1.502(0.785,2.872)$} & \multicolumn{2}{|c|}{$0.758(0.600,0.958)$} \\
\hline$P$-value (log-rank) & \multicolumn{2}{|c|}{$<0.0001$} & \multicolumn{2}{|c|}{0.215} & \multicolumn{2}{|c|}{0.020} \\
\hline
\end{tabular}

Fig. 5 Kaplan-Meier plots of overall survival. a By AFP response, both arms combined. $\mathbf{b}$ By arm, in patients with AFP response. c By arm, in patients with no AFP response. AFP alpha-fetoprotein, $\mathrm{Cl}$ confidence interval, HR hazard ratio, RAM ramucirumab, PBO placebo

setting, AFP response has been evaluated in patients being treated with chemotherapy as well as sorafenib where a response is often associated with a survival advantage. ${ }^{13,20,21}$ In these previously published studies, there were fewer than 200 patients evaluated, only one of them derived data from a randomised study and none was placebo-controlled. Here we report post-hoc analyses of AFP response and progression in 565 patients enrolled in the REACH study. Five hundred and sixty patients with assessable changes in AFP were included in our analysis. An additional advantage of having a placebo arm in our REACH study was to allow assessment of AFP kinetics due to underlying HCC rather than treatment-related. However, in most studies examining systemic therapy, including REACH, the number of patients who experience an AFP response has been quite low. Molecularly targeted agents more commonly result in disease stability, and restricting treatment to patients experiencing an AFP response would exclude a large proportion of patients with stable or slowed progression of AFP levels, who would also derive survival benefit from continued treatment.
In the current analysis of REACH, there was an observed benefit with ramucirumab in delaying time to AFP progression, inducing more frequent and deeper AFP response and lesser AFP progression. AFP changes also correlated with radiographic response and progression. The phase 2 biomarker study of ramucirumab as first-line monotherapy in patients with advanced HCC showed that an AFP decrease was more likely in patients who experienced a radiographic response and an AFP increase more likely in patients with radiographically progressive or nonevaluable disease. ${ }^{22}$ Similar correlations have been made between AFP and other radiographic measures of response with other systemic treatments including sorafenib. ${ }^{13,23-26}$ The observations of changes in AFP and measures of objective response in REACH continue to support a correlation between AFP and objective radiographic measures of tumour assessment.

The results presented here support the notion that the ramucirumab antitumour effect is not restricted to patients with AFP or objective tumour response but rather has some activity in all tumours with varying degree. In the ramucirumab arm, more 
patients experienced both an AFP and a radiographic response compared to placebo. We also observed a shift in the rest of the treated population favouring ramucirumab compared to placebo. More patients in the ramucirumab arm experienced stable AFP or SD compared to placebo. Even in patients who only experienced AFP or radiographic progression, the amplitude of the observed AFP or tumour increase was generally lower.

However, while changes in AFP may correlate with other measures of tumour assessment, neither changes in AFP nor other objective measures of tumour response have been good surrogates to predict $\mathrm{OS}^{27}$ In REACH, while an AFP response was associated with significantly longer OS, analyses support that OS benefit extends to a larger population. Notably, in patients with an elevated baseline AFP $(>1.5 \times$ ULN) a potential OS benefit was still observed in ramucirumab-treated patients compared to placebo, even when AFP responders were excluded; this is likely driven by the much larger proportion of patients who experience disease stability rather than regression. While this re-demonstrates that an elevated baseline AFP can identify the subset of patients most likely to derive an OS benefit, the finding also shows that AFP response is inadequate to select patients most likely to derive a survival benefit. Based on the studies presented here, the lack of an AFP response for a patient should not be used in isolation to judge clinical benefit of systemic treatments like ramucirumab.

Of note, a number of patients on placebo also experienced an AFP response. While the reasons for spontaneous AFP response in the placebo arm are unknown, we note that a similar proportion of patients on the placebo arm also experienced a radiographic response. Other limitations of the results presented here are due to the fact that these were post-hoc analyses performed on a phase 3 study that did not meet its primary end point.

In conclusion, exploratory analyses of REACH show that changes in AFP over time appear to correlate with other measures of objective progression and may help predict patient response, but the utility of AFP to make treatment decisions needs to be validated through a prospective study. Further assessment of the potential benefit of ramucirumab in patients with elevated baseline AFP is being validated in the ongoing $\mathrm{REACH}-2$ study.

\section{ACKNOWLEDGEMENTS}

This study was funded by Eli Lilly and Company. We thank the patients, their families and the study personnel across all sites for participating in this study. Yihuan Xu of Eli Lilly and Company (Bridgewater, NJ) provided statistical expertise. Nathalie Godinot of Eli Lilly and Company (Indianapolis, IN) provided writing assistance. I.C. would like to thank the National Health Service for funding to the National Institute for Health Research Biomedical Research Centre at the Royal Marsden NHS Foundation Trust and The Institute of Cancer Research.

\section{ADDITIONAL INFORMATION}

Supplementary information is available for this paper at https://doi.org/10.1038/ s41416-018-0103-0.

Ethical Approval: Each centre's institutional review board or ethics committee approved the study. The trial followed the principles of the Declaration of Helsinki and the Good Clinical Practice Guidelines of the International Conference on Harmonisation. All patients provided written informed consent.

Competing interests: I.C. reports advisory board roles at Sanofi Oncology, Eli Lilly and Company, Bristol Meyers Squibb, MSD, Bayer, Roche, Five Prime Therapeutics; honoraria from Taiho, Pfizer, Amgen, Eli-Lilly, Gilead Science; research funding from Janssen-Cilag, Sanofi Oncology, Merck-Serono, Novartis. J.O.P. reports receiving clinical research grants, honoraria from Celgene and research grant from AstraZeneca. He reports a role on the advisory board for Celgene. J.-F.B. reports payments from Lilly Oncology, Bayer SP, BMS and Novartis outside the submitted work. M.K. received research grants from Chugai, Otsuka, Takeda, Taiho, Sumitomo Dainippon, Daiichi Sankyo, MSD, Eisai, Bayer, Abbvie, Medico's Hirata, Astellas Pharma and Bristol-Myers Squibb; lecture fees from Bayer, Eisai, MSD, Ajinomoto, Kowa and Taiho; and consulting fees from Kowa, MSD, BMS, Bayer, Chugai, Taiho and Eisai. H.C. C. is a consultant for Eli Lilly and Company, MSD, Merck-Serono, Taiho, BMS, Celltrion, he has received research grants from Eli Lilly and Company, GSK and MSD. J.-M.P. reports receiving research grants from Roche and Merck and payments from Eli Lilly and Company, Bayer, Sanofi, Roche and Merck. T.O. reports research grants, advisory role and honoraria from Eli Lilly, during the conduct of the study; he also reports research grants advisory role and/or honoraria from Novartis Pharma K.K., Kowa K.K., Takeda Bio Development Center Limited, Nippon Boehringer Ingelheim Co., Ltd., Dainippon Simitomo Pharma Co., Ltd., Pfizer Jana Inc, Taiho Pharmaceutical Co., Ltd., Bayer Yakuhin, Ltd., Chugai Pharmaceutical Co., Ltd., Yakuruto Honsha Co., Ltd., Ono Pharmaceutical Co., Ltd., Eisai Co., Ltd, AstraZeneca K.K., Merck Serono Co., Ltd., OncoTherapy Science Inc., Kyowa Hakko Kirin Co., Ltd., Shizuoka Industry, Baxter Nano Carrier Co., Ltd., Zeria Pharmaceutical Co., Ltd., Glaxo Smith Kline K.K. Nobelpharma Co., Ltd., burisutoru, Nipponchemofa, EA Pharma Co., Ltd., FUJIFILM RI Pharma Co., Ltd., Astellas Pharma Inc., Nippon Kayaku Co., Ltd., Daiichi Sankyo Co., Ltd., J-CRSU CO., LTD., outside the submitted work. P.A. and Y.H. are employees of Eli Lilly and Company and own stock from Eli Lilly and Company. A.X.Z. reports that his institution received research support from Eli Lilly and Company. The other authors declare no competing interests.

Note: This work is published under the standard license to publish agreement. After 12 months the work will become freely available and the license terms will switch to a Creative Commons Attribution 4.0 International licence (CC BY 4.0).

Funding: This work was supported by Eli Lilly and Company.

\section{REFERENCES}

1. Ferlay, J. et al. Cancer incidence and mortality worldwide: sources, methods and major patterns in GLOBOCAN 2012. Int. J. Cancer 136, E359-386 (2015).

2. Jelic, S., Sotiropoulos, G. C. \& Group, E. G. W. Hepatocellular carcinoma: ESMO Clinical Practice Guidelines for diagnosis, treatment and follow-up. Ann. Oncol. 21, v59-64 (2010).

3. Gomaa, A. I., Khan, S. A., Leen, E. L., Waked, I. \& Taylor-Robinson, S. D. Diagnosis of hepatocellular carcinoma. World J. Gastroenterol. 15, 1301-1314 (2009).

4. Pons, F., Varela, M. \& Llovet, J. M. Staging systems in hepatocellular carcinoma. HPB (Oxf.) 7, 35-41 (2005).

5. Borzio, M. et al. External validation of the ITA.LI.CA prognostic system for patients with hepatocellular carcinoma: a multicenter cohort study. (HEP-17-0903). Hepatology https://doi.org/10.1002/hep.29662 (2017).

6. Tugues, S., Koch, S., Gualandi, L., Li, X. \& Claesson-Welsh, L. Vascular endothelial growth factors and receptors: anti-angiogenic therapy in the treatment of cancer. Mol. Asp. Med. 32, 88-111 (2011).

7. Amini, A., Masoumi Moghaddam, S., Morris, D. L. \& Pourgholami, M. H. The critical role of vascular endothelial growth factor in tumor angiogenesis. Curr. Cancer Drug Targets 12, 23-43 (2012).

8. Zhu, A. X., Duda, D. G., Sahani, D. V. \& Jain, R. K. HCC and angiogenesis: possible targets and future directions. Nat. Rev. Clin. Oncol. 8, 292-301 (2011).

9. Spratlin, J. L. et al. Phase I pharmacologic and biologic study of ramucirumab (IMC-1121B), a fully human immunoglobulin G1 monoclonal antibody targeting the vascular endothelial growth factor receptor-2. J. Clin. Oncol. 28, 780-787 (2010).

10. Zhu, A. X. et al. Ramucirumab versus placebo as second-line treatment in patients with advanced hepatocellular carcinoma following first-line therapy with sorafenib (REACH): a randomised, double-blind, multicentre, phase 3 trial. Lancet Oncol. 16, 859-870 (2015).

11. Personeni, N. et al. Usefulness of alpha-fetoprotein response in patients treated with sorafenib for advanced hepatocellular carcinoma. J. Hepatol. 57, 101-107 (2012).

12. Memon, K. et al. Alpha-fetoprotein response correlates with EASL response and survival in solitary hepatocellular carcinoma treated with transarterial therapies: a subgroup analysis. J. Hepatol. 56, 1112-1120 (2012).

13. Chan, S. L. et al. New utility of an old marker: serial alpha-fetoprotein measurement in predicting radiologic response and survival of patients with hepatocellular carcinoma undergoing systemic chemotherapy. J. Clin. Oncol. 27, 446-452 (2009).

14. CLIP Investigators. A new prognostic system for hepatocellular carcinoma: a retrospective study of 435 patients: the Cancer of the Liver Italian Program (CLIP) investigators. Hepatology 28, 751-755 (1998).

15. Tangkijvanich, P. et al. Clinical characteristics and prognosis of hepatocellular carcinoma: analysis based on serum alpha-fetoprotein levels. J. Clin. Gastroenterol. 31, 302-308 (2000). 
16. Zhang, X. F. et al. Prognostic factors after liver resection for hepatocellular carcinoma with hepatitis B virus-related cirrhosis: surgeon's role in survival. Eur. J. Surg. Oncol. 35, 622-628 (2009).

17. Wang, N. Y. et al. Prognostic value of serum AFP, AFP-L3, and GP73 in monitoring short-term treatment response and recurrence of hepatocellular carcinoma after radiofrequency ablation. Asian Pac. J. Cancer Prev. 15, 1539-1544 (2014).

18. Riaz, A. et al. Alpha-fetoprotein response after locoregional therapy for hepatocellular carcinoma: oncologic marker of radiologic response, progression, and survival. J. Clin. Oncol. 27, 5734-5742 (2009).

19. Liu, L. et al. The prognostic value of alpha-fetoprotein response for advancedstage hepatocellular carcinoma treated with sorafenib combined with transarterial chemoembolization. Sci. Rep. 6, 19851 (2016).

20. Vora, S. R., Zheng, H., Stadler, Z. K., Fuchs, C. S. \& Zhu, A. X. Serum alphafetoprotein response as a surrogate for clinical outcome in patients receiving systemic therapy for advanced hepatocellular carcinoma. Oncologist 14, 717-725 (2009).

21. Kim, B. et al. Early a-fetoprotein response as a predictor for clinical outcome after localized concurrent chemoradiotherapy for advance $\mathrm{d}$ hepatocellular carcinoma. Liver Int. 31, 369-376 (2011).
22. Zhu, A. X. et al. A phase II and biomarker study of ramucirumab, a human monoclonal antibody targeting the VEGF receptor-2, as first-line monotherapy in patients with advanced hepatocellular cancer. Clin. Cancer Res. 19, 6614-6623 (2013).

23. Matsumoto, Y., Suzuki, T., Ono, H., Nakase, A. \& Honjo, I. Evaluation of hepatoma chemotherapy by alpha-fetoprotein determination. Am. J. Surg. 132, 325-328 (1976).

24. Choi, T. K., Lee, N. W. \& Wong, J. Chemotherapy for advanced hepatocellular carcinoma. Adriamycin versus quadruple chemotherapy. Cancer 53, 401-405 (1984).

25. Chen, L. T. et al. Alpha-fetoprotein response predicts survival benefits of thalidomide in advanced hepatocellular carcinoma. Aliment. Pharmacol. Ther. 22, 217-226 (2005).

26. Lee, S. et al. Early alpha-fetoprotein response predicts survival in patients with advanced hepatocellular carcinoma treated with sorafenib. J. Hepatocell. Carcinoma 2, 39-47 (2015).

27. Llovet, J. M., Villanueva, A., Lachenmayer, A. \& Finn, R. S. Advances in targeted therapies for hepatocellular carcinoma in the genomic era. Nat. Rev. Clin. Oncol. 12, 408-424 (2015). 436 